\title{
Parallel-connected type of Fibonacci Sequence Switched Capacitor DC- DC Converter
}

\author{
Wang Lok Do ${ }^{\mathrm{a},{ }^{*},}$ Kei Eguchi ${ }^{\mathrm{a}}$ \\ ${ }^{a}$ Fukuoka Institute of Technology, 3-30-1 Wajiro-higashi, Higashi-ku, Fukuoka, 811-0295, Japan \\ *Corresponding Author: dwlkiss88@gmail.com
}

\begin{abstract}
The Fibonacci sequence switched capacitor (SC) DCDC converter is a high performance SC DC-DC converter in terms of power efficiency and circuit size among other SC DC-DC converters. However, there is room for improving the power efficiency of the Fibonacci sequence SC DC-DC converter. In this paper, the new topology of the Fibonacci sequence SC DC-DC converter is proposed. The new converter consists of two Fibonacci sequence SC DC-DC converters, and two converters are connected in parallel and across each other. Through this, the proposed converter can reduce internal resistance of it and then the proposed converter can improve power efficiency. To clarify the characteristic of the proposed converter, theoretical analysis and integrated circuit emphasis (SPICE) simulations of the proposed converter are performed.
\end{abstract}

Keywords: DC-DC converter, Fibonacci sequence, switched capacitor.

\section{Introduction}

With the importance of energy harvesting system, photovoltaic system, electric vehicles, hybrid vehicles and so on, the power converter of those devices is required to have high power efficiency and small size. For this reason, switched capacitor (SC) DC-DC converter was suggested.

An SC DC-DC converter consists of switches and capacitors; the switches are semiconductor switch like metaloxide semiconductor field-effect-transistor (MOSFET) or transistor. The first idea of SC DC-DC converter was proposed as $\mathrm{SC}$ voltage multiplier ${ }^{(1)}$. From the first idea, many types of the SC DC-DC converter were suggested and designed. Among others, the Fibonacci sequence SC DC-DC converter can meet the demand of high power efficiency and small size. It was confirmed in previous researches that the Fibonacci sequence SC DC-DC converter has the highest power efficiency and the smallest size among voltage equational type, series-parallel type, ring type, multistage switched capacitor voltage multiplier ${ }^{(2)-(6)}$. However, there is room for improving the Fibonacci sequence SC DC-DC converter about power efficiency and operating state.

The conventional Fibonacci sequence SC DC-DC converters operated by using three states: two charging states and one discharging state. The extra discharging state of the conventional converter makes the converter's impedance higher. In this situation, we suggest the new topology of Fibonacci sequence SC DC-DC converter in this paper. The new topology has two states for operating it by connecting two Fibonacci sequence SC DC-DC converters in parallel and across each other. Through this, the proposed converter can operate by two states, which means that internal resistance of the converter is decreasing and improve the power efficiency of it.

The rest of this paper is organized as follows. In chapter 2 , the circuit configuration of the conventional and the proposed Fibonacci sequence SC DC-DC converter is presented. In chapter 3 , the comparison between the conventional and the proposed Fibonacci sequence SC DCDC converter is presented by the theoretical the analysis. In chapter 4, the characteristic of the proposed converter is clarified by SPICE simulation. Finally, conclusion and future study are shown in chapter 5 .

\section{Circuit Configuration}

\subsection{Conventional Fibonacci Sequence SC DC-DC converter}

Fig. 1 shows the conventional Fibonacci sequence DCDC converter. The converter consists of 10 transistor 


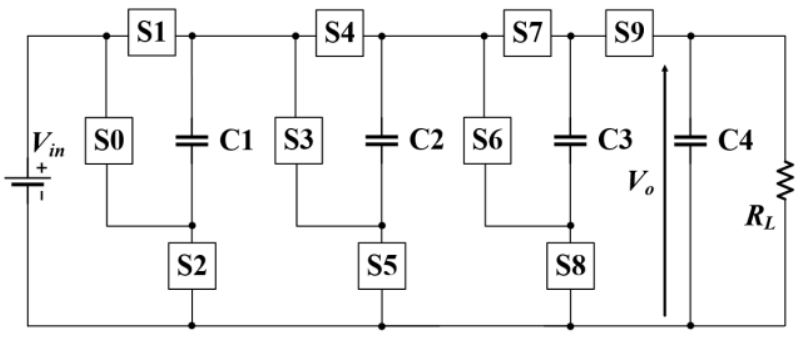

Fig. 1. Conventional Fibonacci sequence SC DC-DC converter.

Table 2. Switching rule of the conventional Fibonacci sequence SC DC-DC converter.

\begin{tabular}{|c|c|c|}
\hline Step & Switch to turn on & Switch to turn off \\
\hline State-1 & $S 0, S 4, S 5$ & Others \\
\hline State-2 & $S 1, S 2, S 3, S 7, S 8$ & Others \\
\hline State-3 & $S 1, S 3, S 6, S 9$ & Others \\
\hline
\end{tabular}

switches and 4 capacitors. The converter operate by the switching rule as shown on table 1 . The reason that the converter's title has Fibonacci is that the capacitors in the converter are charged by the following equation (1).

$$
\begin{aligned}
& V_{C n}=V_{C n-1}+V_{C n-2} \text { where } n=\{3\} \\
& \text { with } V_{c 1}=V_{i n} V_{c 2}=V_{c 1}+V_{i n}
\end{aligned}
$$

In State-1, the converter charges the even number capacitor (C2) up to 2 times of the input voltage. In State-2, the odd number capacitors $(\mathrm{C} 1, \mathrm{C} 3)$ are charged up to 1 times and 3 times of the input voltage respectively. In State-3, the charged capacitors ( $\mathrm{C} 2$ and $\mathrm{C} 3$ ) and the input source generate 6 times stepped-up voltage. In other words, State-1 and -2 are for charging the capacitors and State- 3 is for discharging the capacitors' energy to the output. Fig. 2 shows the waveforms of the current and voltage for each state.

\subsection{Proposed Fibonacci sequence SC DC-DC converter}

The idea of the proposed Fibonacci sequence SC DCDC converter was starting from room for improving the conventional converter as following. 1) A possibility to decrease the number of the operating states. 2) A possibility to improve the power efficiency of the converter. The

Table 2. Switching rule of the conventional Fibonacci sequence SC DC-DC converter.

\begin{tabular}{|c|c|c|}
\hline Step & Turn on & Turn off \\
\hline State-1 & $S 0, S 4, S 5, S 9, S 8, A 1, A 2, A 3, A 6, A 7$ & Others \\
\hline State-2 & $A 0, A 4, A 5, A 9, A 8, S 1, S 2, S 3, S 6, S 7$ & Others \\
\hline
\end{tabular}

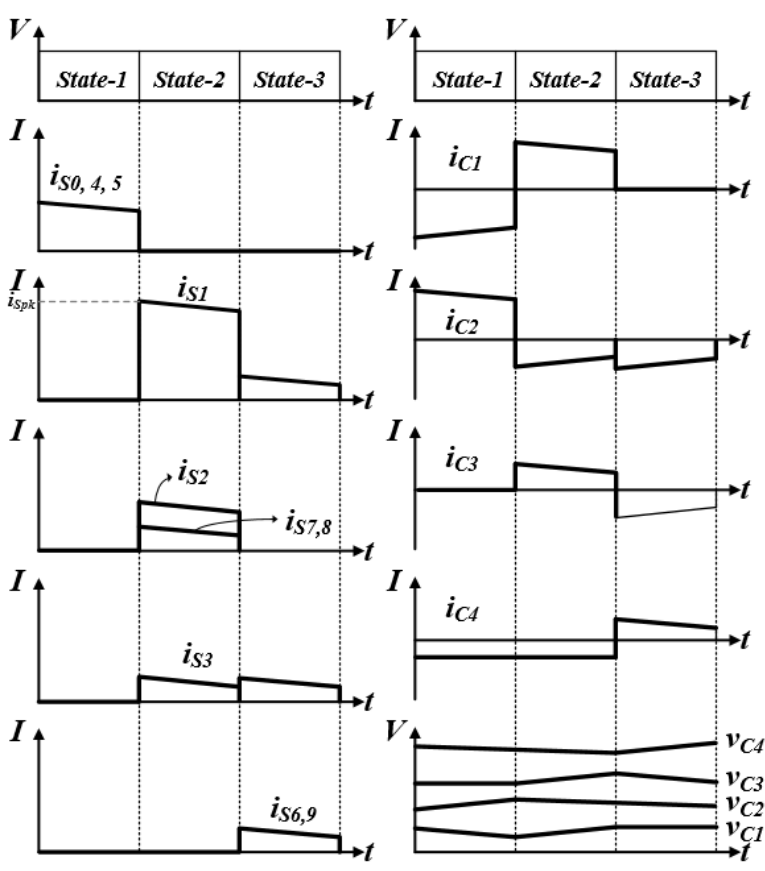

(a)

(b)

Fig. 2. Waveforms of Conventional Fibonacci sequence SC DC-DC converter. (a) Current of switches.

(b) Current and voltage of capacitors.

solution is to remove some switches of the conventional converter and then to connect two converters in parallel and cross. In this way, it is possible to operate the proposed converter without the state- 3 of the conventional converter.

The proposed converter consists of two conventional Fibonacci sequence SC DC-DC converter with removing the switch S6 in the conventional converter as shown fig. 1. The two converters are connected in parallel and cross by using four transistors switch as shown fig 3 .

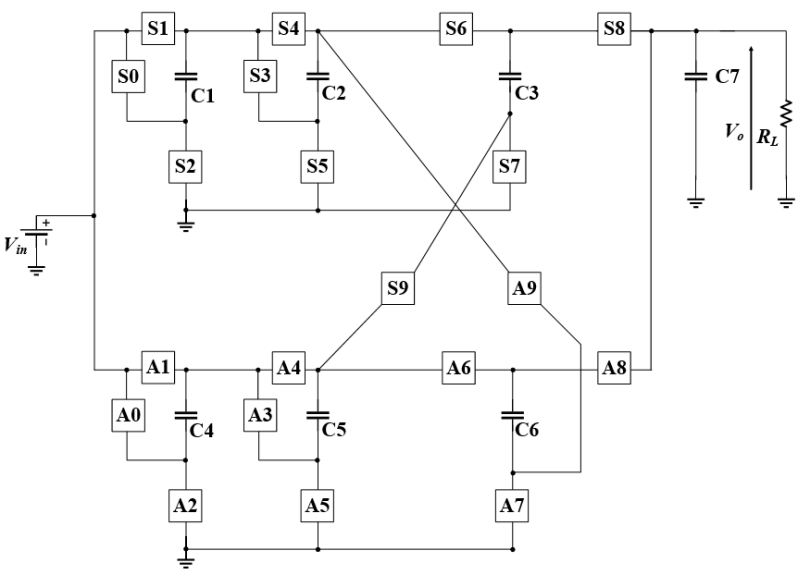

Fig. 3. Proposed Fibonacci sequence SC DC-DC converter. 

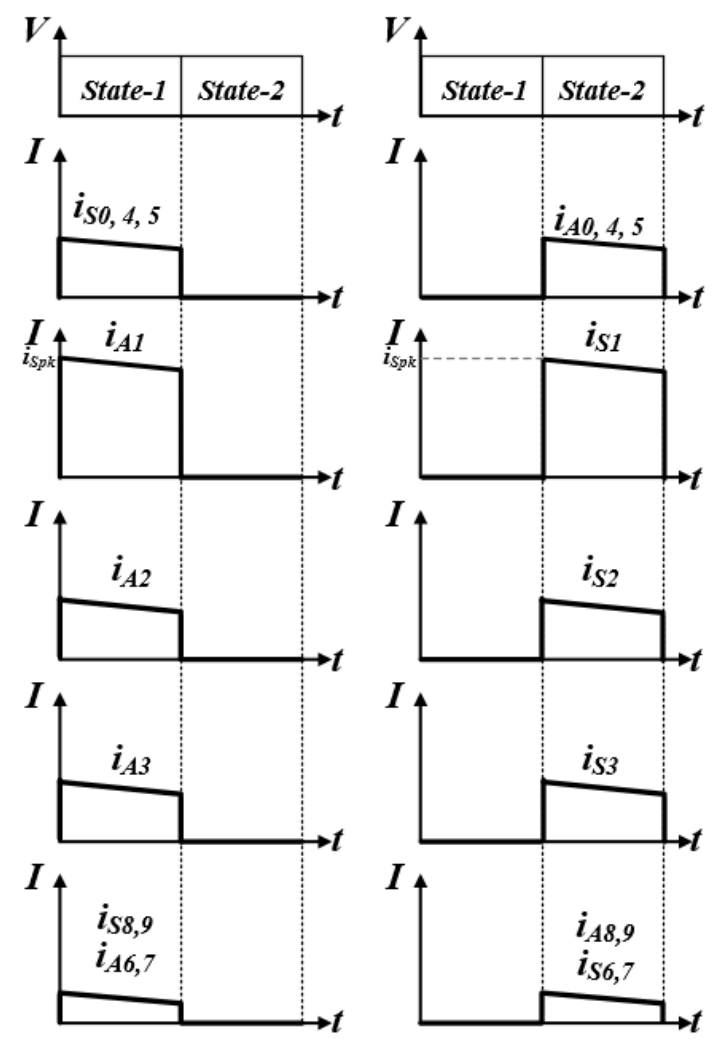

(a)
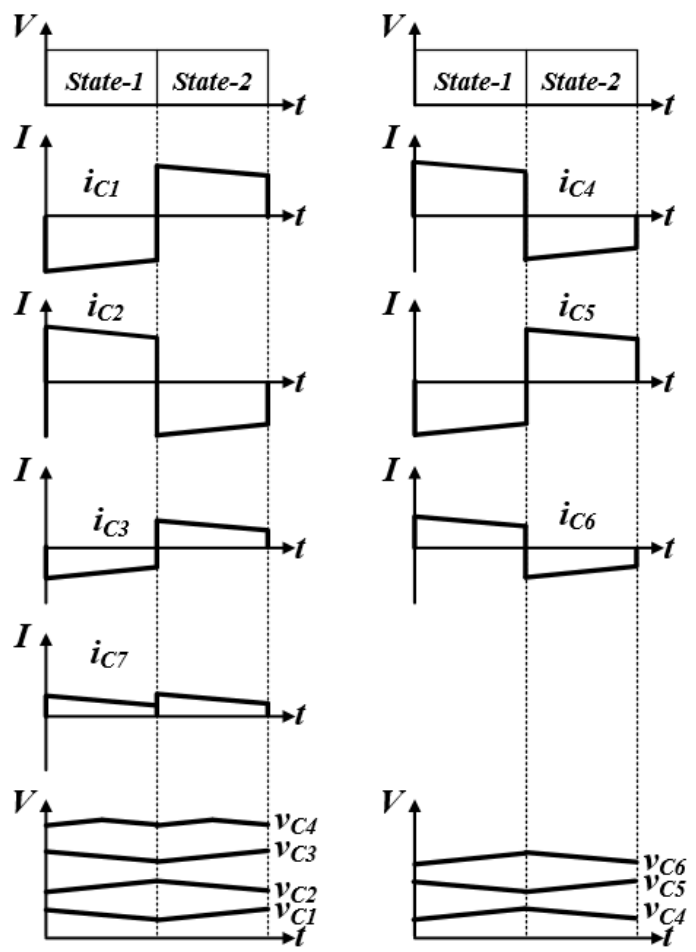

(b).

Fig. 4. Waveforms of proposed Fibonacci sequence SC DC-DC converter. (a) Current of switches. (b) Current and voltage of capacitors.

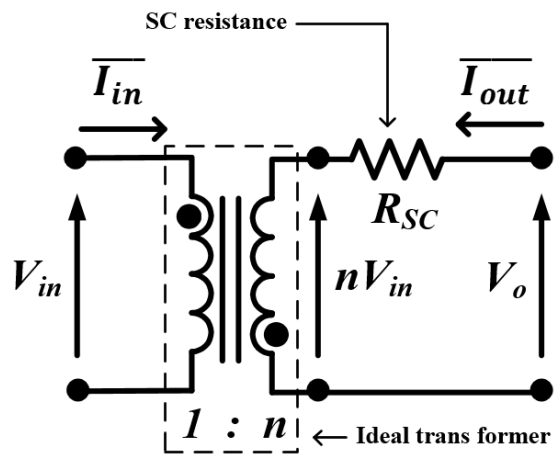

Fig. 5. Four-terminal equivalent model for the converter in this paper.

Unlike the conventional converter of fig. 1, the proposed converters operated by two states as shown on table 2. For State-1and -2 , each cell of the proposed converter charges and discharges such as fig. 4. Each cell's charging way is the same as that of the conventional converter as above-mentioned. In a steady state, the proposed converter has $\mathrm{C} 3$ and $\mathrm{C} 6$ charged to 3 times of the input voltage respectively. C3 and C6 can generate 6 times stepped-up voltage to connect two capacitor's node in series, which means that it is not necessary to use the extra operating state for discharging like State-3 of the conventional converter. Namely, the proposed converter can operate charging and discharging at the same time.

\section{Theoretical Analysis}

\subsection{Equivalent model}

To analyze theoretically the converters, the fourterminal equivalent model is used. Fig. 5 describes the model, where $n$ means the ratio of conversion and $R_{s c}$ is the switched capacitor resistance. The value of $R_{s c}$ is only one component to consume power from the input source in the model. Therefore, the value of $R_{s c}$ has influence on power efficiency of the converter. By using circuit laws and $R_{s c}$ value, it is implemented to derive the power efficiency, the relation with the input current and the output current and the relation with the input voltage and the output voltage in the theoretical analysis.

In the theoretical analysis, we assume two things as follows. 1) All switches in the converters are an ideal switch with on-resistance $R_{o n}$. 2) Time constant related with capacitor and resistance is much larger than the system clock of the converters.

3.2 Theoretical analysis of the conventional Fibonacci 


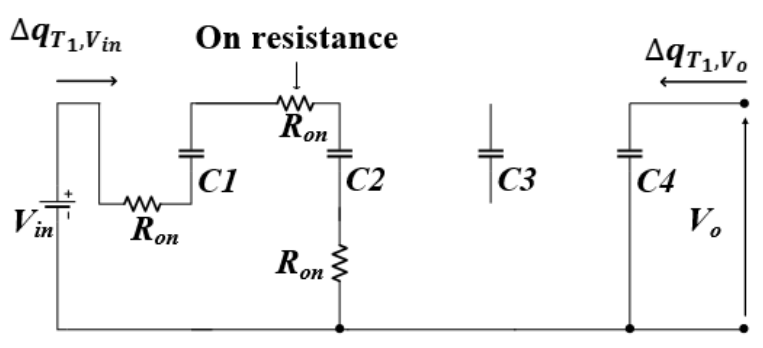

(a) State-1.

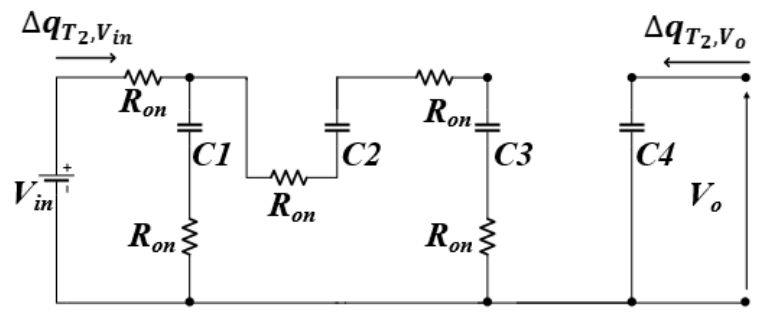

(b) State-2.

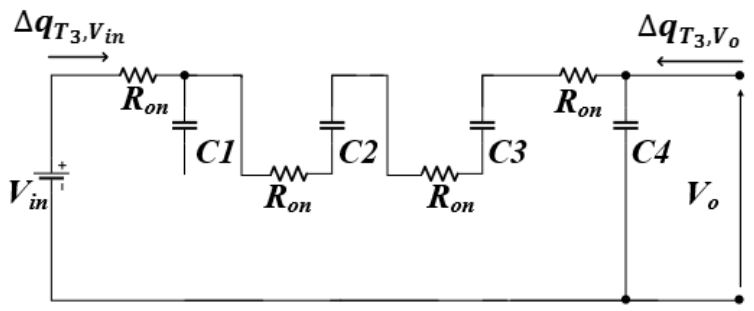

(c) State-3

Fig. 6. Instantaneous equivalent circuit of conventional converter at each state

\section{Sequence SC DC-DC converter}

In the theoretical analysis of the conventional converter, the interaction relations are based on the instantaneous equivalent circuits shown fig. 6 .

In a steady state, all of the differential value of the electric charge satisfy equation (2).

In equation (2), $\Delta q_{T i}{ }^{k}$ means that the electric charge of the $k$-th capacitor in State- $i$, and $T$ is the total period of the system clock for State-1, 2 and 3. Additionally, $T_{i}$ is the $i$-th period of the State- $i$. By applying the Kirchhoff's current law to the each circuit in fig 6 , the interaction relations can be derived. Equations (3) - (5) are for State-1, 2 and 3 respectively.

$$
\begin{gathered}
\Delta q_{T_{1}, V_{i n}}=-\Delta q_{T_{1}}^{1}, \Delta q_{T_{1}}^{3}=0 \\
\text { and } \Delta q_{T_{1}, V_{o}}=\Delta q_{T_{1}}^{4} \\
\Delta q_{T_{2}, V_{i n}}=\Delta q_{T_{2}}^{1}-\Delta q_{T_{2}}^{2}, \Delta q_{T_{2}}^{2}=-\Delta q_{T_{2}}^{3} \\
\text { and } \Delta q_{T_{2}, V_{o}}=\Delta q_{T_{2}}^{4} \\
\Delta q_{T_{3}, V_{i n}}=-\Delta q_{T_{3}}^{2}, \Delta q_{T_{3}}^{1}=0 \\
\text { and } \Delta q_{T_{2}, V_{o}}=\Delta q_{T_{3}}^{4}+\Delta q_{T_{3}}^{3}
\end{gathered}
$$

By using the equations (3) - (5), the average value of the input current and output current can be derived as the equation (6).

$$
\begin{gathered}
\overline{I_{l n}}=\frac{\sum_{i=1}^{3} \Delta q_{T_{i}, V_{i n}}}{T}=\frac{\Delta q_{T, V_{i n}}}{T} \\
\text { and } \\
\overline{I_{o}}=\frac{\sum_{i=1}^{3} \Delta q_{T_{i, V_{o}}}}{T}=\frac{\Delta q_{T, V_{o}}}{T}
\end{gathered}
$$

By substituting equations (2) - (5) into equation (6), the relation between the input current and the output current can be obtained as equation (7).

$$
\begin{gathered}
\overline{I_{l n}}=-6 \overline{I_{o}} \\
\text { where } \Delta q_{T_{i}, V_{i n}}=-6 \Delta q_{T, V_{o}} \text { and } n=6
\end{gathered}
$$

Next, the calculation of the consumed energy for each state is necessary to derive the value of $R_{s c}$. The total consumed energy can be expressed as equation (8) by using equations (2) - (5).

$$
\begin{gathered}
W_{T}=\sum_{i=1}^{3} W_{T_{i}}=\frac{96 R_{\text {on }}}{T}\left(\Delta q_{V_{o}}\right)^{2}, \\
\text { where } W_{T_{1}}=\frac{12 R_{\text {on }}}{T_{1}}\left(\Delta q_{V_{o}}\right)^{2}, \\
W_{2}=\frac{16 R_{\text {on }}}{T_{2}}\left(\Delta q_{V_{o}}\right)^{2} \text { and } \\
W_{3}=\frac{4 R_{\text {on }}}{T_{3}}\left(\Delta q_{V_{o}}\right)^{2}
\end{gathered}
$$

The consumed energy of the equivalent model shown in fig. 5 is able to express as equation (9).

$$
\begin{gathered}
\sum_{i=1}^{3} \Delta q_{T_{i}}^{k} \text { where } T=T_{1}+T_{2}+T_{3} \\
\text { and } T_{1}=T_{2}=T_{3}=\frac{T}{3} . \\
W_{T}=\left(\frac{\Delta q_{V_{o}}}{T}\right)^{2} \cdot R_{S C} \cdot T
\end{gathered}
$$

By comparing equation (8) and (9), the value of $R_{s c}$ is obtained as equation (10).

$$
R_{S C}=96 R_{o n}
$$

The power efficiency $\eta$ and the output voltage $V_{o}$ are 
can be obtained as equation (11) by using the value of $R_{s c}$ and the equivalent model.

$$
\begin{gathered}
\eta=\frac{R_{L}}{R_{L}+96 R_{\text {on }}} \text { and } \\
V_{o}=\left(\frac{R_{L}}{R_{L}+96 R_{\text {on }}}\right) \times 6 V_{\text {in }}
\end{gathered}
$$

\subsection{Theoretical analysis of the proposed Fibonacci Sequence SC DC-DC converter}

The interaction relations of the proposed converter are based on the instantaneous equivalent circuits shown in fig. 7.

In steady state, all of the differential value of the electric charge satisfy equation (12).

$$
\begin{aligned}
& \sum_{i=1}^{2} \Delta q_{T_{i}}^{k} \text { where } T=T_{1}+T_{2} \\
& \text { and } T_{1}=T_{2}=\frac{T}{2} .
\end{aligned}
$$

Each parameter of $\Delta q_{T_{i}}{ }^{k}$ is used in the same way as above-mentioned at section 3.2. The interaction relations for each equivalent circuit in fig. 7 can be derived as equation (13) and (14) by using Kirchhoff's current law.

$$
\begin{gathered}
\Delta q_{T_{1}, V_{i n}}=-\Delta q_{T_{1}}^{1}+\Delta q_{T_{1}}^{4}-\Delta q_{T_{1}}^{5} \\
\Delta q_{T_{1}}^{3}=-\Delta q_{T_{1}}^{2}, \Delta q_{T_{1}}^{5}=-\Delta q_{T_{1}}^{6}+\Delta q_{T_{1}}^{3} \\
\text { and } \Delta q_{T_{1}, V_{o}}=\Delta q_{T_{1}}^{7}+\Delta q_{T_{1}}^{3} \\
\Delta q_{T_{2}, V_{i n}}=-\Delta q_{T_{2}}^{4}+\Delta q_{T_{2}}^{1}-\Delta q_{T_{2}}^{2} \\
\Delta q_{T_{2}}^{4}=-\Delta q_{T_{2}}^{5}, \Delta q_{T_{2}}^{2}=-\Delta q_{T_{2}}^{3}+\Delta q_{T_{2}}^{6} \\
\text { and } \Delta q_{T_{1}, V_{o}}=\Delta q_{T_{2}}^{7}+\Delta q_{T_{2}}^{6}
\end{gathered}
$$

The average value of the input current and output current can be derived as the equation (15) by using equations (13) and (14).

$$
\begin{gathered}
\overline{I_{l n}}=\frac{\sum_{i=1}^{2} \Delta q_{T_{i}, V_{i n}}}{T}=\frac{\Delta q_{T, V_{i n}}}{T} \\
\text { and } \\
\overline{I_{o}}=\frac{\sum_{i=1}^{2} \Delta q_{T_{i}, V_{o}}}{T}=\frac{\Delta q_{T, V_{o}}}{T}
\end{gathered}
$$

By substituting equations (13) and (14) into equation (15), we can confirm that the relation between the input current and the output current is same as equation (7).

Next step is the calculation of the consumed energy for

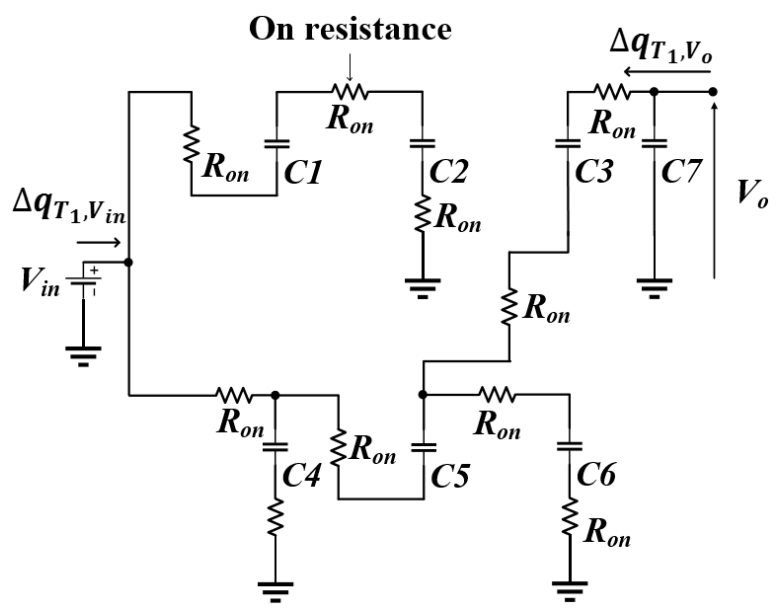

(a) State-1.

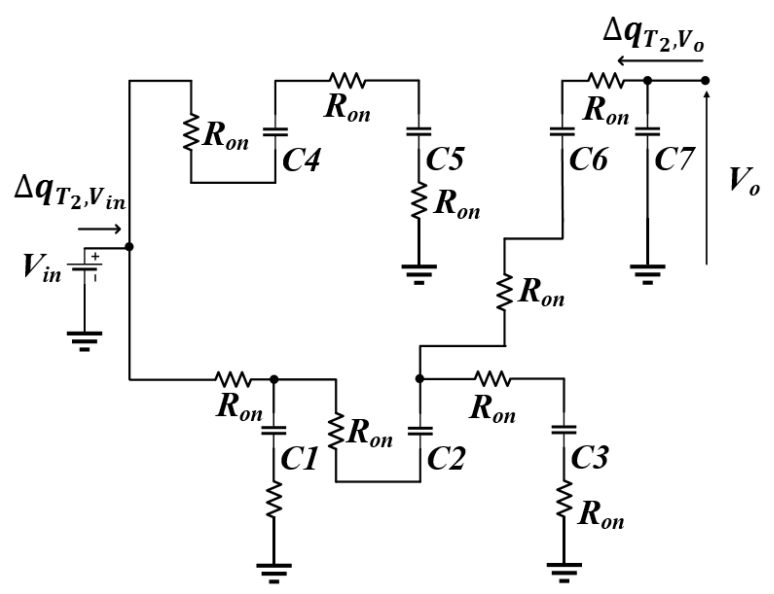

(b) State-2.

Fig. 7. Instantaneous equivalent circuit of proposed converter at each state

each state in order to derive the value of $R_{s c}$. The total consumed energy can be expressed as equation (16) by using equations (12) - (14).

$$
\begin{gathered}
W_{T}=\sum_{i=1}^{2} W_{T_{i}}=\frac{20 R_{\text {on }}}{T}\left(\Delta q_{V_{o}}\right)^{2}, \\
\text { where } W_{T_{1}}=W_{2}=\frac{10 R_{\text {on }}}{T_{1}}\left(\Delta q_{V_{o}}\right)^{2}
\end{gathered}
$$

By comparing equation (9) and (16), the value of $R_{s c}$ is obtained as equation (17).

$$
R_{S C}=20 R_{o n}
$$

The power efficiency $\eta$ and the output voltage $V_{o}$ are can be derived as equation (18) by using the value of $R_{s c}$ and the equivalent model. 
Table 3. Value of $R_{s c}$ and the number of circuit components

\begin{tabular}{|c|c|c|}
\hline Parameter & Conventional & Proposed \\
\hline$R_{s c}$ & $96 R_{o n}$ & $20 R_{o n}$ \\
\hline Capacitors' umber & 5 & 7 \\
\hline Switches' number & 10 & 18 \\
\hline
\end{tabular}

Table 4. Simulation conditions

\begin{tabular}{|c|c|}
\hline Parameter & Value \\
\hline$V_{\text {in }}$ & $12 \mathrm{~V}$ \\
\hline Capacitance & $10 \mu \mathrm{F}$ \\
\hline$R_{\text {on }}$ & $0.1 \Omega$ \\
\hline$R_{L}$ & $10 \Omega \sim 10 \mathrm{k} \Omega$ \\
\hline$T$ & $1.5 \mu \mathrm{sec}$ \\
\hline
\end{tabular}

$$
\begin{gathered}
\eta=\frac{R_{L}}{R_{L}+20 R_{o n}} \text { and } \\
V_{o}=\left(\frac{R_{L}}{R_{L}+20 R_{o n}}\right) \times 6 V_{i n}
\end{gathered}
$$

\subsection{Comparison between the conventional converter} and the proposed converter

Through the theoretical analysis, we can know that the value $R_{s c}$ of the proposed converter has 4.8 times lower than that of the conventional converter. It means that the proposed converter can improve the power efficiency of it. Table 3 shows the value of $R_{s c}$ and the number of circuit component respectively.

\section{Simulation}

To clarify the characteristics of the converters, the proposed and the conventional converter are simulated with the conditions as table 4. Additionally, the reason that the range of load resister starts from $10 \Omega$ is to simulate the performance of the converters at the worst power efficiency. As you can see from fig. 8, the proposed converter offers higher output voltage than the conventional converter. The reason for the difference of output voltage of two converters is each power efficiency. Fig. 9 demonstrates that the proposed converter improves the power efficiency over whole output power by comparing the conventional converter. Concretely, the power efficiency of the proposed converter can improve about $30 \%$ higher than the conventional converter when the output power is about $130 \mathrm{~W}$.

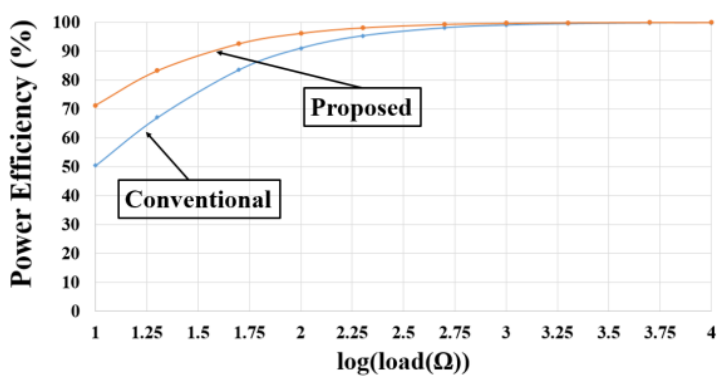

(a) Efficiency as the load increased.

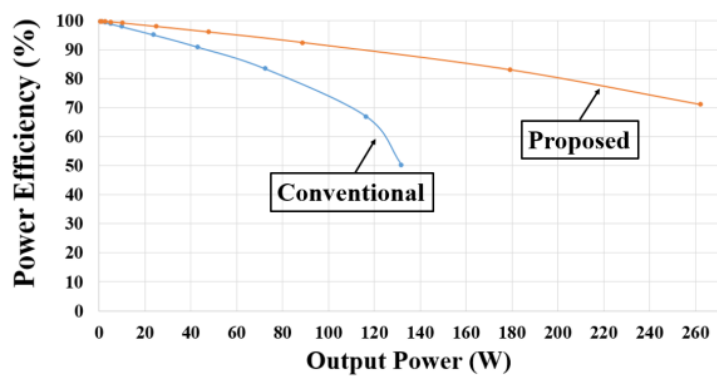

(b) Efficiency as the output power increased.

Fig. 9. Simulated and theoretical power efficiency.

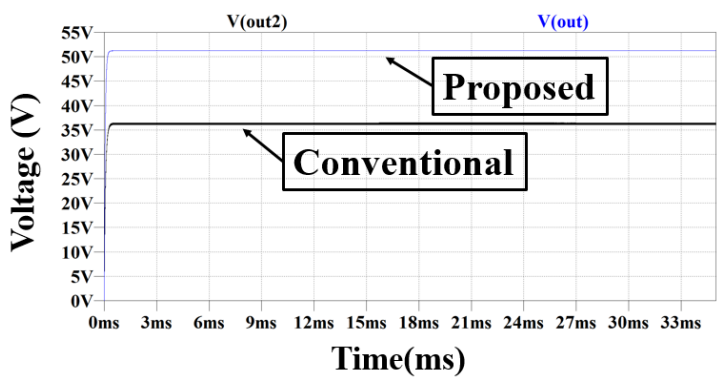

(a) $R_{L}=10 \Omega$

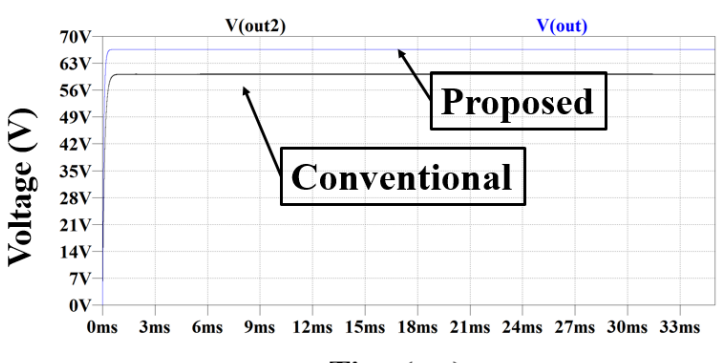

Time(ms)

(b) $R_{L}=50 \Omega$.

Fig. 8. Simulated output voltage with the load.

\section{Conclusion}

A new topology of Fibonacci sequence SC DC-DC converter has been proposed in this paper. The proposed SC DC-DC converter can operate by using two states, whereas the conventional Fibonacci sequence SC DC-DC converter operates by three states. While the circuit size of the 
conventional converter is smaller than the proposed converter, we confirmed theoretically that the proposed converter has higher power efficiency. The result of the simulations showed that the proposed converter operated with higher power efficiency than the conventional converter. The proposed converter can operate with the maximum $30 \%$ higher power efficiency at about $130 \mathrm{~W}$ of the output power than the conventional converter.

The experiment of the proposed converter are left as a future study in order to confirm the validity of it.

\section{References}

(1) J. S. Brugler : "Theoretical performance of voltage multiplier circuits", IEEE Journal of Solid-State Circuits, Vol. 6, Issue:3, pp.132-135, 1971.

(2) M. Oota, S. Terada, K. Eguchi, and I. Oota : "Development of switched-capacitor bi-directional DCAC converter for inductive and capacitive loads", IEEE International Symposium on Industrial Electronics, pp. 1618-1623, 2009

(3) S. Terada, I. Oota, K. Eguchi, and F. Ueno : "A ring type switched-capacitor (SC) programmable converter with DC or AC input/ DC or AC output", the 47th IEEE International Midwest Symposium on Circuits and Systems, pp. I-29 - I-32, 2004

(4) Y. H. Chang : "Modelling and analysis of multistage switched-capacitor-voltage-multiplier boost DC-AC inverter", IEEE 9th International Conference on Power Electronics and Drive Systems, pp. 523-526, 2011

(5) L. Salem, P. Mercier : “A 45-ratio recursively sliced series-parallel switched-capacitor DC-DC converter achieving 86\% efficiency", Proc. of IEEE Custom Integrated Circuit Conference 2014, pp. 1-4, 2014

(6) K. Eguchi, K. Abe, W. Do : "Synthesis and analysis of a simple inductor-less DC-AC inverter", Proc. of 2016 6th International Workshop on Computer Science and Engineering, pp. 315-319, 2016 